Contents lists available at www.ijpba.in

International Journal of Pharmaceutical and Biological Science Archive

PubMed (National Library of Medicine ID: 101738825)

Index Copernicus Value 2017: 71.80

Volume 7 Issue 1; January-February; 2019; Page No. 01-05

\title{
CYBERKNIFE ROBOTIC RADIOSURGERY SYSTEM
}

\author{
Gupta Saibya, Gupta Priya \\ Department of Pharmaceutical Science, Banasthali Vidyapith, Jaipur, Rajasthan, India.
}

\begin{abstract}
The latest addition to the CyberKnife product family, which was released in September 2009. This review updates the previous technical reviews of the original system version published in the late 1990s. There is a clear dose response for localized prostate cancer radiotherapy and there probablyis a radiobiological rationale for hypo-fractionation. Combining the two should maximize tumor control and increase the therapeutic ratio. Technical developments over the last decade have impacted virtually every aspect of the CyberKnife System. These developments have increased the geometric accuracy of the system and have enhanced the dosimetric accuracy and quality of treatment, with advanced inverse treatment planning algorithms, rapid Monte Carlo dose calculation, and post-processing tools that allow trade-offs between treatment efficiency and dosimetric quality to be explored. The CyberKnife can produce superior DVHs for sparing of rectum and bladder and excellent DVHs for target coverage compared with IMRT, and possesses dose heterogeneities to the same degree as IMRT plans.
\end{abstract}

Keywords: CyberKnife System; Radiosurgery; Stereotactic Body Radiation Therapy; Hypofractionation; IMRT Hetrogeneities

\section{INTRODUCTION}

The CyberKnife ${ }^{\circledR}$ Robotic Radiosurgery System has undergone nearly twenty years of technical development from its conception [1] to its most current type, the CyberKnife VSITM System, the first of which was installed in April 2010. The CyberKnife System, as described in a series of technical papers in the late 1990s (27), created as a frameless alternative to prevailing stereotactic radiosurgery systems such as the Gamma Knife and conventional linear accelerators (LINACS) prepared with head frames and stereotactic smile collimators The CyberKnife, is not only limited to, benign and malignant primary tumors, brain metastases, trigeminal neuralgia, auditory neuromas and arteriovenous malformations (AVMs).

CyberKnife radiosurgery is even used to treat complicated neurosurgical cases, while sparing important functions, such as hearing and vision. The key to CyberKnife ${ }^{\circledR}$ surgery is the delivery of the right dose of radiation to a very precise area whereas minimising the damaging effects on normal tissue.

The CyberKnife is unique in that it uses a compact linear accelerator (LINAC) mounted on an image-guided robotic arm to deliver multiple beams of high energy $x$-rays to a target.

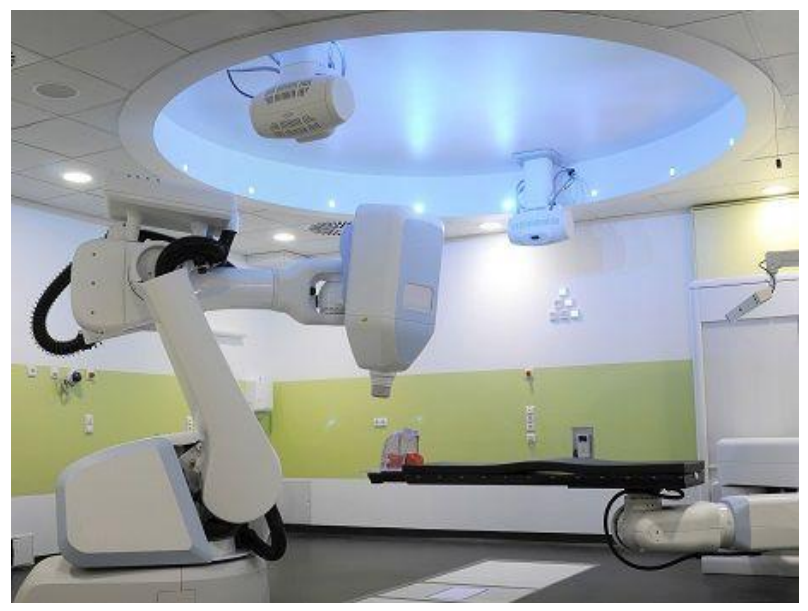

Figure 1: Cyberknife Instrument

\section{HISTORY:}

The Cyberknife was first permitted by the FDA for intracranial application in 1999, and then 
conventional full-body clearance in 2001. Since its beginning the CyberKnife has treated in excess of 120,000 patients, at over 230 centers around the world. Doctors have used radiosurgery since the 1950s, the CyberKnife was invented in 1987 by John R. Adler M.D., a Stanford University Medical Center professor of Neurosurgery and Stereotactic Radiosurgery. The CyberKnife combines two systems: a compact, unimportant linear accelerator mounted on a robotic arm that sends the radiation to the patient and an image guidance system that tracks the tumor's location within the body in real time to direct the radiation to the precise location where it is needed and adjusting the beam within millimeter tolerance up to 100 or more times during each treatment session. In fact, it is the only system that can right according to the information given while following tumor position continually during treatment. The robotic arm that delivers the radiation is so maneuverable that physicians may treat a lesion from up to 1600 non-coplanar aiming angles. This means they may use it to treat tumors and other injuries anywhere in the body without open surgery.[8]

\section{Medical research on cyberknife:}

The Medical Staff of the Artemis CyberKnife Center comprises a group of Doctors, Medical Physicists, and scientists devoted to cancer care, research, and patient attention. The CyberKnife Center Medical Staff is dynamically complex in a distinction of research projects on the suggestions and efficiency of CyberKnife treatments. The CyberKnife Center's Medical Staff includes an Internal Review Board and a directive to join in radical Clinical Trials. [9]

Table 1:

\begin{tabular}{|c|c|c|}
\hline & $\begin{array}{l}\text { Cyberknife System in 1997- } \\
1999\end{array}$ & Cyberknife VSI system in 2010 \\
\hline $\begin{array}{l}\text { Image Registration and tracking } \\
\text { method }\end{array}$ & $\begin{array}{l}\text { Skull skeletal tracking with 3D } \\
\text { translation correction }\end{array}$ & $\begin{array}{l}\text { 1. Skull skeletal tracking with } 6 \mathrm{~d} \text { translational and } \\
\text { rotation corrections } \\
\text { 2. Spine skeletal tracking with } 6 \mathrm{D} \text { correction } \\
\text { 3. Fiducial marker tracking with } 6 \mathrm{D} \text { correction }\end{array}$ \\
\hline Robotic manipulator precision & $0.55 \mathrm{~mm}$ & $0.12 \mathrm{~mm}$ \\
\hline $\begin{array}{l}\text { Overall targeting accuracy (static } \\
\text { target) }\end{array}$ & $\begin{array}{l}\text { Mean }: 1.6 \mathrm{~mm} \\
\text { Range: } 0.6-2.5 \mathrm{~mm}\end{array}$ & Maximum: $<=0.95 \mathrm{~mm}$ \\
\hline Dose calculation algorithm (s) & Ray tracing & $\begin{array}{l}\text { 1. Monte-carlo } \\
\text { 2. Ray tracing }\end{array}$ \\
\hline Robot path traversal & Robot moves through all & Robot moves through modes \\
\hline Clinical applications & $\begin{array}{l}\text { Intracranial and upper spine } \\
\text { radio surgery }\end{array}$ & $\begin{array}{l}\text { Anywhere in the body where radio surgery is } \\
\text { clinically indicated. }\end{array}$ \\
\hline Total patients treated to date & 30 & 90,000 \\
\hline
\end{tabular}

\section{Treatment procedure:}

The CyberKnife one of the most advanced forms of radiosurgery is a easy, non-invasive treatment that distributes high doses of precisely targeted radiation to abolish tumors or injuries within the body. It uses a robotic arm to deliver greatly focused beams of radiation.

The tractability of the robotic arm marks it possible to treat areas of the body such as the spine and spinal cord, that can't be frozen by other radiosurgery techniques. UCSF Medical Center, which originated offering CyberKnife treatments in 2003, is one of only a few midpoints in California that offers this service.

\section{Procedure:}

Team Member: CyberKnife System team includes:

- A thoracic surgeon 
- A radiation oncologist

- An interventional radiologist

- A medical physicist

- A radiation therapist

- A dosimetrist

- Medical support staff

\section{Preparation:}

As part of the treatment process, doctors will recognize the location and size of the tumor. Depending on the outcomes, the CyberKnife System will be able to use the recognizing characteristics of the tumor itself to clearly envisage the tumor treatment. Some tumors may require the placement of fiducials within the lung. Fiducials are markers that show on $x-$ ray to help the CyberKnife System isolate the tumor's exact location. They are implanted in a short outpatient procedure prior to treatment planning. If fiducials are required, the patient must wait approximately one week before Cyberknife treatment .As part of the preparation process, patients will be fixed for a special body cradle designed to make treatment more comfortable and to ensure body position is the same for each treatment session. The patient also will be fitted with a special vest, which is worn during CyberKnife treatment and enables the robot to associate chest motion and breathing patterns with the tumor position.

While lying in the cradle, a CT scan will be performed to determine the exact size, shape and location of the tumor. A MRI or PET scan also may be necessary to fully envision the tumor and nearby anatomy.

\section{Treatment Plan}

Using the imaging data, your medical team will determine the size of the area being targeted by radiation, the radiation dose, and identify critical structures - such as the spine or nearby organs - where radiation should be minimized. The CyberKnife System then calculates the optimal radiation delivery plan, leveraging its extreme maneuverability to deliver a safe and accurate treatment.

\section{Treatment}

During treatment, the CyberKnife System's computer-controlled robot will change around your body to many positions from which it will deliver radiation. At each position, it will stop to determine precisely where the radiation would be delivered by associating breathing motion with the tumor. The CyberKnife Organization then corrects to ensure the optimum radiation delivery plan, leveraging its life-threatening maneuverability to distribute a safe and truthful treatment.

\section{Post-treatment}

Most patients knowledge minimal side effects and can fast return to their daily routines with little break to their normal activities. Doctors will discuss all probable side effects former to treatment and may prescribe medication to control any side effects.[16-17]

Most common sites include:

- Benign tumor

- Brain tumor

- Colon tumor

- Head and Neck tumor

- Kidney

- Liver

- Lung

- Metastasis

- Pancreas

- Prostate

Application:

$>$ It is the treatment of choice in cases where further surgery is not feasible, like in inaccessible lesions, lesions lying in close proximity with critical structures, and irregularly shaped tumors, as it spares the adjacent normal cells.

$>$ It is used in Intercranial conditions to radiosurgically treat a variety of tumours such as menigiomas, acoustic neuromas, pituitary tumours, and metastases as well as other abnormalities such as arteriovenous malformations (AVMs)

$>$ It is indicated as the line of treatment for head and neck cancers.

$>$ It is used as an excellent procedure in the treatment of trigeminal neuralgia. Other 
were used to treat intracranial lesions, but with recent advancements in technology, CyberKnife has emerged as a whole-body stereotactic radiosurgery system.

$>$ It is also an indication for extracranial tumors, and small tumors in the lung, liver, spine and pancreas.[19-21]

\section{Advantages:}

Non-invasive and pain-free radiosurgical treatment is now available to patients.

$>$ It is now possible to treat tumours of the spine, pancreas, lung and liver safely and effectively with radiosurgical

$>$ The newest development in Cyberknife radiosurgery is a breath-triggered realtime movement- correction system, which makes it possible to apply radiosurgical doses to these lesions.

$>$ Anaesthesia or dedicated body stereotactic frames, as used with conventional radiation devices to suppress respiration, are no longer necessary

$>$ A hospital stay or rehabilitation is not needed in most cases.

$>$ Radiosurgery course lasts between 60 and 90 minutes.

\section{Limitation:}

- It is a technique-sensitive procedure. So, it involves a team of radiation oncologists, medical physicists, and technologists for the successful outcome of treatment.

- Re-irradiation has been associated with harmful complications such as necrosis, focal edema, and hemorrhage, while no such complications have been encountered in patients who underwent only radiosurgical procedure.[25]

- As CyberKnife treatment involves delivery of high dose of radiations, negligence can result in death of patient.[26-28]

- $\quad$ Longer treatment time, i.e. more than 40 min, may pose a problem in pediatric patients, where adequate sedation and immobilization is needed, and in elderly patients suffering from respiratory disorders and bladder insufficiency.

\section{Conclusion:}

Cyberknife has proved to be an accurate, safe, superior, and sophisticated approach for the treatment of intracranial and extracranial lesions. In dentistry, I is being successfully used to treat oral cancer and trigeminal neuralgia. The technological advances in stereotactic neurosurgery will show the way for further extension in treatment. New research activities are aimed at utilizing the full potential of robotics in radiosurgery and also on the prospective enduring side effects. By use of an optimized path traversal process, the overall treatment time is reduced by $5-10 \mathrm{~min}$. Monte Carlo algorithms have been used to calculate the accurate dose required. The IRIS collimator decreases the treatment time, requires less monitor units, and provides more homogeneity of treatment. Fiducial-less treatment is possible now with the use of real-time image guidance. Treatment of depression, obsessive compulsive disorder, Parkinsonism, non-small cell lung tumor, etc. by Cyberknife is still under research. Another aim is to treat low-volume tumors.

\section{References:}

1. S. L., the Cyberknife: a frameless robotic system for radiosurgery. Stereotact Funct Neurosurg 69, 124-128 (1997).Adler, J. R., Schweikard, A., Murphy, M. J., Hancock, S. L. Image-

2. Guided cyberknife radiosurgery. In: Barnett, G., Roberts, D., Maciunas, R. J. (eds) Image-Guided Neurosurgery: Clinical Applications of Interactive Surgical Navigation, edn. Quality Medical Publishing, pp 193-204 (1998).Adler, J. R., Jr., Murphy, M. J., Chang, S. D., Hancock, S. L. Image

3. Guided robotic radiosurgery. Neurosurgery 44, 1299-1306; discussion 1306-1297 (1999).Murphy, M. J., Cox, R. S. The accuracy of dose localization for an

4. Image-guided frameless radiosurgery system. Med Phys 23, 2043-2049 (1996). Murphy,

5. M. J. An automatic six-degree-of-freedom image registra. tion algorithm for imageguided frameless stereotaxic radiosurgery. 
A., Bodduluri, M., Adler, J. R. Planning for camera-

6. Guided robotic radiosurgery. IEEE Transactions on Robotics and Automation 14, 951-962 (1998).

7. http://aboutcancer.com/radiosurgery.htm

8. http://www.cyberknifedelhi.com/historyof-the-cyberknife

9. http://www.cyberknifedelhi.com/medicalresearch-on-cyberKnife

10. Adler, J. R., Jr., Chang S. D., Murphy M. J., Doty J., Geis P., Hancock 2. S. L., The Cyberknife: a frameless robotic system for radiosurgery. Stereotact Funct Neurosurg 69, 124-128 (1997)

11. Adler, J. R., Schweikard, A., Murphy, M. J., Hancock, S. L. Image-3. guided cyberknife radiosurgery. In: Barnett, G., Roberts, D., Maciunas, R. J. (eds) Image-Guided Neurosurgery: Clinical Applications of Interactive Surgical Navigation, edn. Quality Medical Publishing, pp 193-204 (1998).

12. Adler, J. R., Jr., Murphy, M. J., Chang, S. D., Hancock, S. L. Image-4. guided robotic radiosurgery. Neurosurgery 44, 1299-1306; discussion 1306-1297 (1999).

13. Murphy, M. J., Cox, R. S. The accuracy of dose localization for an 5 . image-guided frameless radiosurgery system. Med Phys 23, 2043-2049 (1996).

14. Murphy, M. J. An automatic six-degree-offreedom image registra6. tion algorithm for image-guided frameless stereotaxic radiosurgery. Med Phys 24, 857-866 (1997).

15. Schweikard, A., Bodduluri, M., Adler, J. R. Planning for camera-7. guided robotic radiosurgery. IEEE Transactions on Robotics and Automation 14, 951-962 (1998).

16. https://www.cyberknife.com/treatment/proc ess/lung
17. https://cyberknife.com/treatment/process/ge neral

18. https://www.cyberknifetampabay.org/disea ses-treated-by-cyberknife.

19. Muacevic A, Wowra B. CyberKnife radiosurgery - A new treatment method for image-guided, robotic, high-precisio radiosurgery. Eur Neurol Rev 2007;32-3.

20. Yamazaki $H$, Ogita $M$, Himei $K$, Nakamura $S$, Yoshida K, Kotsuma $\mathrm{T}$, et al. Hypofractionated stereotactic radiotherapy using CyberKnife as a boost treatment for head and neck cancer, a multi institutional survey: Impact of planning target volume. Anticancer Res 2014;34:5755-9.

21. Joseph B, Supe SS, Ramachandra A. CyberKnife: A double edged sword? Rep Pract Oncol Radiother 2010;15:93-7.

22. Kurup G. CyberKnife: A new paradigm in radiotherapy. J Med Phys 2010;35:63-4.

23. Chang SD, et al. In: Radiosurgery, Kondziolka D (ed.), New York: Karger Medical and Scientific Publishers, 1999

24. Schweikard A, Shiomi H, Adler Jr JA, American Association of Physicists in Medicine, 2006;(31):2738-

25. Joseph B, Supe SS, Ramachandra A. CyberKnife: A double edged sword? Rep Pract Oncol Radiother 2010;15:93-7.

26. Srivastava $R$, Jyoti $B$, Gupta $M$, Singh $N$. CyberKnife ${ }^{\circledR}$ : The cu $\square$ ing edge technology in precision surgery. SRM J Res Dent Sci 2015;6:106-15.

27. Kodani N, Yamazaki H, Tsubokura $T$, Shiomi $\mathrm{H}$, Kobayashi $\mathrm{K}$, Nishimura $\mathrm{T}$, et al. Stereotactic body radiation therapy for head and neck tumor: Disease control and morbidity outcomes. J Radiat Res2011;52: 24-31

28. Eshleman JS, Berkenstock KG, Singapuri KP, Fuller C. The CyberKnife ${ }^{\circledR}$ M6TM radio surgery system. J Lanc Gen Hosp 2013;8:44-9. 